\title{
Experiencias de padres de niños ciegos: un camino con grandes desafios
}

\section{Parent experiences with blind children: a very challenging path}

\author{
Liliana Cristina Morales Viana ${ }^{1}$
}

Forma de citar: Morales Viana LC. Experiencias de padres de niños ciegos: un camino con grandes desafíos. Rev Univ Ind Santander Salud. 2016; 48(3): 331-340. DOI: http://dx.doi.org/10.18273/revsal.v48n3-2016007 @) (1) Ð

\section{RESUMEN}

Introducción: La discapacidad es un tema complejo, de enorme repercusión social y económica. Una de las discapacidades más devastadora es la visual, dado que conlleva graves efectos psico-sociales, especialmente si se presenta en niños, por que afecta la calidad de vida de ellos y la de sus familias. Cuando se tiene un niño con limitación visual, se origina un duelo similar al que se produce por la pérdida de un ser querido. Existen pocos estudios relacionados con las vivencias de los padres de niños con discapacidad visual. Objetivo: Conocer las experiencias y sentimientos de los padres ante la discapacidad visual de sus hijos. Metodología: Estudio cualitativo. La metodología utilizada fue la fenomenología interpretativa. La muestra estuvo conformada por siete participantes, seis mujeres y un hombre, cuidadores familiares de niños con diagnóstico de ceguera, matriculados en el Instituto de Niños Ciegos y Sordos del Valle del Cauca, a quienes se les realizaron tres entrevistas en profundidad. Resultados: Los participantes vivieron múltiples desafíos, desde un embarazo inesperado, la hospitalización del pequeño, la devastadora noticia del diagnóstico, el proceso de aceptación y la convivencia con la discapacidad visual de sus hijos; ante estos desafíos los participantes experimentaron ira, tristeza, miedo, culpa, incertidumbre y vergüenza. Conclusión: La discapacidad visual representa una carga para los niños y sus familias, es un tema complejo que debe ser abordado desde equipos interdisciplinarios. Para la enfermera constituye un desafío, pues el cuidado se debe centrar en ayudar al niño ciego a utilizar al máximo su capacidad residual. El objetivo principal es lograr que el niño y su familia vivan un proceso de adaptación a la discapacidad visual lo menos traumático posible.

Palabras Clave: Discapacidad, ceguera, experiencias, padres.

\begin{abstract}
Introduction: Disability is a very complex issue of great social an economic impact. One of the most devastating disabilities is visual impairment, as it leads to serious psychosocial effects, especially in the cases of children, affecting the quality of life for them and their families. When there is a child with visual disability, it can produce similar effects as mourning felt after the loss of a dear relative. There are few studies related to the parents experiences of children with visual impairment. Objective: To document the experiences and feelings of parents with visually impaired children. Methodology: Qualitative study. The methodology used was the interpretative

1. Universidad del Valle. Cali, Colombia

Correspondencia: Liliana Cristina Morales Viana. Dirección: Calle 14 No 83-21 Unidad Residencial Riveras del Faro. Barrio El Ingenio II. Correo electrónico liliana.morales@correounivalle.edu.co. Teléfono: 3165318574.


phenomenology. The sample was made of seven participants, six women and one man, the primary caregivers of children diagnosed with blindness, enrolled at the Institute for Blind and Deaf Children of Valle del Cauca, who were interviewed in depth 3 times. Results: Participants experienced multiple challenges, from an unexpected pregnancy, the childs hospitalization, the devastating news of the diagnosis, the process of accepting and living with visual disabilities of their children; to these challenges participants experienced anger, sadness, fear, guilt, shame uncertainty Conclusion: Visual impairment is a burden both the children and their families and is a complex issue that must be addressed from interdisciplinary teams. For nurses is a challenge, because care must focus on helping the blind child to use the most of their functional residual capacity. The main objective is to support the child and his family to live an adaptation process to the visually impaired as smooth as possible.

Keywords: Disability, blindness, experiences, parents.

\section{INTRODUCCIÓN}

La Organización Mundial de la Salud (OMS) define la discapacidad como las deficiencias, las limitaciones en la actividad y las restricciones en la participación ${ }^{1}$. La discapacidad es un tema complejo, de enorme repercusión social y económica, pero del que existen pocos datos. Los estudios estadísticos son escasos ${ }^{2}$. La discapacidad es un fenómeno que afecta al $10 \%$ de la población mundial, en Latinoamérica se reportan 85 millones de personas con discapacidad, para el caso de Colombia el censo poblacional realizado en el 2005 reportó que el $6,3 \%$ de la población presenta alguna limitación permanente ${ }^{3}$.

Una de las discapacidades más devastadora es la visual, dado que conlleva graves efectos psico-sociales; se estima que en el mundo hay aproximadamente 39 millones de personas con discapacidad visual, de los cuales 1.4 millones son niños ( $0-14$ años $)^{4}$, el $60 \%$ de estos niños mueren antes del 1er año del diagnóstico de ceguera, el 90\% de la carga mundial de discapacidad visual se concentra en los países en desarrollo5.

La ceguera infantil produce un profundo impacto en los niños, dado que altera el neurodesarrollo, lo que afecta la calidad de vida de los niños y sus familias, además los "años ceguera" son más costosos en niños, porque cuando sean adultos va a ser muy difícil asegurarles un empleo remunerado, por lo que deberán usar los recursos económicos de su familia y del sistema de salud ${ }^{4}$.

En los Estados Unidos, las causas de ceguera en niños son: discapacidad visual cortical, hipoplasia del nervio óptico y la retinopatía del prematuro $(\mathrm{ROP})^{4}$, para Latinoamérica, los países como Argentina, Colombia, Cuba, Ecuador, Guatemala, México y Paraguay, comparten como principal causa de ceguera en los niños, la ROP. Para Colombia la ROP representa un $33.8 \%$ sobre las otras causas ${ }^{6,7}$.
La atención en salud a las personas que presentan discapacidad y sus familias, es compleja, requiere del trabajo de equipos multidisciplinarios con sólida formación disciplinar, para el caso de enfermería, el aporte podría ser realizar el cuidado desde un paradigma más abarcador, que intente comprender a la persona desde sus vivencias, pero además, teniendo en cuenta que la principal causa de ceguera en niños es la retinopatía del prematuro, la enfermera puede participar en la prevención de ésta, a través de acciones de cuidado específicas para evitar su desarrollo.

Cuando se tiene un niño con limitación visual, se origina un duelo similar al que viven las personas cuando se produce la pérdida de un ser querido. El duelo más crítico para los padres es la aceptación de un niño no imaginado ni esperado ${ }^{8}$. Existe $n$ pocos estudios relacionados con las vivencias de los padres de niños con discapacidad visual, por lo cual se realizó el presente estudio con el propósito de contribuir al conocimiento científico sobre la vida de los padres de niños ciegos. El estudio tuvo como objetivo conocer las experiencias, y sentimientos de los padres ante la discapacidad visual de sus hijos. El conocimiento de las vivencias de los padres puede contribuir a que el profesional de enfermería y de salud en general, provea una atención a los padres teniendo en cuenta su mundo vivencial, lo cual les puede ayudar a adaptarse a la discapacidad de sus hijos, y generarles el menor sufrimiento posible a los niños y a sus familias.

Este estudio fenomenológico mostró que los padres experimentaron ira, tristeza, miedo, incertidumbre y vergüenza, ante la discapacidad visual de sus niños. Se recomienda a los profesionales del equipo multidisciplinario, responsable del cuidado de los padres y niños ciegos, tener en cuenta estos hallazgos para lograr una atención integral que satisfaga las necesidades y expectativas de los mismos. 


\section{MATERIALES Y MÉTODOS}

Diseño del estudio: el estudio es de carácter cualitativo. La metodología empleada fue la fenomenología interpretativa basada en la filosofía de Heidegger, que tiene como objetivo comprender las experiencias cotidianas y articular las similitudes y las diferencias en los significados, compromisos, prácticas, habilidades y experiencias de los seres humanos 9 . Por lo anterior, es apropiada para estudiar las experiencias de los cuidadores primarios de niños ciegos.

Muestra: los participantes fueron cuidadores familiares de niños con diagnóstico de ceguera, matriculados en el Instituto de Niños Ciegos y Sordos del Valle del Cauca. La muestra estuvo conformada por siete participantes, seis mujeres y un hombre, cinco madres, una abuela y un padre, con edades comprendidas entre 18 y 45 años. El tamaño de muestra fue determinado usando la técnica de la saturación de la información, que se alcanza cuando el investigador obtienen la misma información o similar, pues lo informantes no indican algo diferente de lo ya dicho ${ }^{10}$.

Criterios de selección: los participantes cumplieron los siguientes criterios de inclusión: Ser el cuidador familiar de un niño ciego, estudiante del Instituto de Niños Ciegos y Sordos del Valle del Cauca y cuyas edades estuvieran entre los 6 y 10 años de edad (la muestra fue a conveniencia, pues se consideró que los padres de los niños con estas edades, tenían mayor experiencia, y podrían ofrecer información más detallada sobre el fenómeno de interés); el cuidador debía tener más de 18 años de edad, y convivir con el niño en la misma casa. Se denomina cuidador familiar a la persona que tiene un vínculo de parentesco o cercanía y asume la responsabilidad del cuidado de un ser querido que vive con enfermedad crónica y participa en la toma de decisiones, y supervisa y apoya la realización de las actividades de la vida diaria para compensar la disfunción existente en la persona con la enfermedad ${ }^{10}$. Se excluyeron cuidadores diagnosticados con alguna alteración psiquiátrica (diagnosticada por psiquiatra, y que estuviera en el registro del niño en la institución).

Plan de recolección de la información: en un día programado, la investigadora hizo contacto con los cuidadores con el fin de explicarles acerca de la investigación. Además, en este contacto se obtuvo la firma del consentimiento informado.

La investigadora realizó tres entrevistas en profundidad a cada uno de los participantes, con una duración aproximada de una hora, con un intervalo de tiempo entre las entrevistas de un mes. Las dos primeras entrevistas fueron individuales y se llevaron a cabo en la institución, brindando privacidad a los participantes. La última entrevista se realizó en el hogar de los participantes. En la primera entrevista se incluyeron aspectos para determinar la percepción que tenían los participantes ante la situación de discapacidad de sus hijos y los temores que ellos experimentaron. Se permitió que los participantes expresaran libremente sus experiencias de tener un hijo con discapacidad visual. En la Segunda entrevista fue acerca de las expectativas que tenían los participantes para el futuro del hijo discapacitado y las vivencias que como padres experimentaron frente al proceso de adaptación a la discapacidad de sus hijos. Para la última entrevista, se realizó una visita domiciliaria.

La investigadora grabó y transcribió textualmente las entrevistas. La investigadora tiene experiencia en estudios cualitativos y en el cuidado de enfermería a niños y adolescentes. El análisis de los datos se desarrolló paralelamente a la recolección de datos, inicialmente se hizo un análisis superficial especialmente para reconocer aspectos que debían ser ampliados en las siguientes entrevistas, al finalizar la recolección de la información se hizo un análisis profundo, que consistió en leer y releer las entrevistas, con el fin de descubrir las unidades de análisis, luego se hizo la codificación de las unidades, que posteriormente constituyeron los temas que se presentan en los resultados.

El estudio fue aprobado por el Comité de Ética de La Facultad de Salud de la Universidad del Valle y del Instituto de Niños Ciegos y Sordos del Valle del Cauca. Se tuvo en cuenta la coherencia ó credibilidad, aplicabilidad y auditabilidad de los $\operatorname{resultados}^{12}$. A continuación se describen las estrategias empleadas por la investigadora para garantizar el rigor del estudio según estos criterios.

Coherencia o credibilidad: se demostró cuando los participantes reconocieron la descripción de los resultados como parte de la realidad vivida por ellos. Para atender a este criterio, la investigadora presentó a cada uno de los participantes en el estudio las descripciones de los resultados, y se les pidió que verificasen si las mismas correspondían a lo que ellos habían expresado. Los participantes reconocieron las descripciones como propias. Aplicabilidad (validez externa): implica que los resultados tengan significado para otros en situaciones similares. Se espera que los hallazgos del estudio puedan ser aplicados a otros padres que viven situaciones similares. Auditabilidad: 
en la presente investigación la recolección de la información y la interpretación o análisis fue un proceso cuidadoso. La descripción detallada de los aspectos metodológicos del estudio y las características de los informantes y su proceso de selección, procedimiento para recolectar y analizar la información permiten que otros investigadores conozcan y evalúen la metodología y los resultados del estudio.

Durante el desarrollo de la investigación se garantizó la confidencialidad de los participantes. A cada cuidador se le asignó un código el cual usó para codificar la transcripción de las entrevistas. La información digital se guardó en un computador con acceso restringido por clave de seguridad. Las grabaciones se mantuvieron en sobres sellados y archivados con acceso solo para la investigadora. La información obtenida solo se uso para la investigación.

\section{RESULTADOS}

A. Caracterización de los participantes en el estudio:

Tabla 1. Caracterización participantes en el estudio "Experiencias de padres de niños ciegos: un camino con grandes desafíos" Cali 2016

\begin{tabular}{ccccc}
\hline $\begin{array}{c}\mathbf{N}^{\mathbf{0}} \text { DE } \\
\text { PARTICIPANTE }\end{array}$ & PARENTESCO & $\begin{array}{c}\text { EDAD EN } \\
\text { ANOS }\end{array}$ & $\begin{array}{c}\text { ESCOLARIDAD DEL } \\
\text { PARTICIPANTE }\end{array}$ & DIAGNÓSTICO DEL NIÑO \\
\hline 1 & Padre & 30 & Bachillerato completo & Retinopatía del prematuro \\
2 & Abuela & 45 & Primaria completa & Retinopatía del prematuro \\
3 & Madre & 18 & Bachillerato incompleto & Retinopatía del prematuro \\
4 & Madre & 22 & Bachillerato incompleto & Retinopatía del prematuro \\
5 & Madre & 27 & Bachillerato completo & Toxoplasmosis congénita \\
6 & Madre & 28 & Bachillerato completo & Toxoplasmosis congénita \\
7 & Madre & 38 & Bachillerato incompleto & Retinopatía del prematuro \\
\hline
\end{tabular}

Fuente: Morales Liliana. Caracterización de los participantes en el estudio "Experiencias de padres de niños ciegos: un camino con grandes desafíos" Cali 2016

B. Temas centrales: surgieron cuatro temas en los que se reflejan las experiencias de los cuidadores ante la discapacidad visual de los niños. A continuación se presentan:

\section{La experiencia de la gestación}

Para la mayoría de las participantes el embarazo fue inesperado, una de las participantes relató haberse enterado del embarazo cuando tenía veinte semanas debido a que su periodo menstrual era normal y el motivo de consulta fue el aumento exagerado de peso. Otra de las madres expresó que comenzó a presentar el crecimiento de las mamas, lo que produjo la sospecha del embarazo.

Ante lo inesperado del embarazo no todos los padres recibieron la noticia con alegría, ellos también tuvieron sentimientos como incertidumbre, tristeza, algunas sintieron miedo pues en ese momento de sus vidas no estaban preparadas para un embarazo. Una de las participantes expresó que solo deseaba tener un hijo, otra manifestó que estaba muy joven y no era el momento de tener un bebé, otra de las participantes creía que debía esperar hasta que tuviera una buena situación económica, otra por el contrario deseaba tener un hijo pero por presentar diabetes e hipertensión no podría embarazarse por el riesgo. Sin embargo ante la 334 noticia del embarazo, lo asumieron con responsabilidad, por lo tanto, en el transcurso de los meses estuvieron pendientes del desarrollo de su hijo. Sintieron emoción al percibir los movimientos fetales, escuchar los latidos de su corazón, observar las ecografías.

Yo estaba estudiando y quede embarazada, el papá de mi hijo era mi primer novio, fue algo que no esperaba, me di cuenta a los dos meses, no había planeado el embarazo estaba nerviosa, porque yo quede joven en embarazo, por eso me tuve que casar. Después de esto, todos en la casa aceptaron el embarazo, estaba feliz por el niño" Madre No 1.

Para las participantes fue importante asistir a los controles prenatales, ellas siguieron las recomendaciones del equipo de salud como: comer sanamente, no fumar, ni ingerir bebidas alcohólicas, para tener un embarazo sin complicaciones. Sin embargo, la mayoría de las participantes después de haber transcurrido las dieciocho semanas de gestación debieron consultar al médico por presentar síntomas como: fiebre, malestar general, edema en miembros inferiores, prurito, vómito, aumento anormal de peso lo que les generó estrés y preocupación por lo que podía ocurrir con la vida de sus pequeños. 
Los participantes tuvieron la sensación que hubo negligencia por parte del equipo de salud, dado que las complicaciones del embarazo pudieron haberse prevenido o controlado, si se hubieran realizado exámenes de laboratorio más oportunos, previniendo así el nacimiento prematuro, el avance de las patologías del embarazo que como consecuencia produjeron la ceguera de sus niños.

"A mi me dio tres días fiebre y yo creo que fue en ese momento donde se activo la toxoplasmosis, los doctores decían vete para la casa, tómate el dólex, mucho liquido y reposo. Uno se confía y me decian no vayas a tomar nada más. Cuando el infectólogo me vio el decía que lo habian tratado mal, debieron mandarte exámenes para ver qué pasaba. Él me dijo que si me hubieran hecho los exámenes hubieran podido prevenir muchos daños, y la niña no estaría ciega" Madre No 2

\section{La llegada del bebe: jel parto se hizo inminente!}

Para los participantes fue difícil conocer que sus hijos y nieta debían nacer prematuros. Ellos deseaban que el embarazo fuera a término o por lo menos que durara unas semanas más para que su bebe no fuera tan pequeño, describieron que fueron momentos angustiosos cuando les informaron que debían realizarles una cesárea, tenían miedo, temblaban y rezaban, sólo una de las participante no se dio cuenta del proceso dado que estaba inconsciente.

La angustia y la intranquilidad no solo fue de las madres y padres que esperaban por su hijo, también de otros miembros de la familia como abuelas, tíos y hermanos. En la sala de espera, de las instituciones donde atendieran a la madre, la familia estaba a la expectativa de la vida del binomio madre e hijo, sabían que el parto era de riesgo y que tanto el recién nacido como la madre estaban en peligro, además se les había informado que no podrían ver al bebé dado que debía ser trasladado a la unidad de cuidados intensivos.

"Vi que paso un enfermero con una cosa pequeñita llena de tubos, una enfermera me dijo esa que llevan ahí, es su nieta. Yo estaba angustiada pues debía dejar a la niña en la incubadora porque era muy pequeña, transparente y se le veían las venas, le faltaba tiempo." Abuela

Durante el embarazo y el parto los padres vivieron momentos difíciles, sin embargo mantuvieron la esperanza que una vez terminara lo relacionado con el nacimiento del bebé y la recuperación de la madre, sus vidas retornarían a la normalidad. Esto no fue así, tanto las madres como los niños debieron quedarse hospitalizados.

Las participantes refieren que el proceso de recuperación de la cesaría fue difícil pues no solo era el dolor físico, sino la tristeza y la incertidumbre por estar separadas de sus hijos, ellas se sentían solas, y una de las participantes manifestó sentirse abandonada, pues su esposo y familia estuvieron más pendientes del recién nacido.

"Yo lo único que lograba enfocar era que la prioridad era Valentina, olvide a mi esposa, pero igual si hubiese existido la atención de un psicólogo yo hubiese podido dar apoyo a mi señora, yo solo iba un momentico y me iba para donde la niña" Padre

\section{La experiencia de la hospitalización y el impacto del diagnóstico, el principal desafío}

Después de vivir lo relacionado con el nacimiento, las participantes debieron enfrentar la hospitalización de los pequeños, la cual inicialmente la percibieron como algo necesario, pero no dejaron de sentir tristeza por no poder tenerlos a su lado, no poder amamantarlos y brindarles el amor y la protección de padres. Ellos habían soñado vivir el proceso que toda familia tiene, que al salir del hospital podrían llevar sus hijos en brazos, pero debían quedarse y el cuidado dependía del equipo de salud.

"Tenia miedo de dejar a mi hija en la incubadora, quería tenerla a mi lado y llevármela para la casa, eso es algo terrible, pues una sueña con tener a su hija, compartir con ellos desde que se levanta hasta que anochece" Madre No 3

Otro aspecto importante que generó impacto a los padres, fue la apariencia física de los pequeños, pues a diferencia del niño idealizado se encontraron con un niño muy pequeño, delgado, velludo, sin uñas, muy pálido. Los participantes experimentaron sentimientos de desilusión y miedo.

Debido a la hospitalización y la prematurez, los niños vivían constantes procedimientos como transfusiones, punciones para exámenes de laboratorio, monitoria permanente, oxigenoterapia, terapia respiratoria y su alimentación era a través de catéteres o por sondas. Los múltiples procedimientos generaron en los padres miedo, tristeza, impotencia, angustia y llanto dado que percibían los procedimientos como fuentes de sufrimiento y dolor para sus pequeños. 
Los participantes percibieron que el tiempo para la visita de sus bebés fué muy reducido, esta situación los hacía sentirse distantes de sus hijos, y con sentimientos de pérdida de su rol de padres, ellos siempre desearon mayores oportunidades de interacción con el recién nacido. Todas las participantes expresaron que les hizo mucha falta el poder amamantar a sus bebes, para ellas la lactancia era una forma de brindar amor y protección a sus pequeños y al no hacerlo se sentían culpables, sintieron como si los hubiesen abandonado.

Otro aspecto importante con el que debieron lidiar los participantes, fue que debieron vivir con la idea de la muerte de sus pequeños, el médico tratante les informó la gran probabilidad que tenían los niños de morir, frecuentemente imaginaban el día que llamaran de la clínica para informarles que su bebé había muerto. El contacto con otros padres de niños en iguales o peores condiciones que la de sus hijos, les aumentaba el miedo y la incertidumbre, especialmente con padres a quienes se les morían sus pequeños.

"Yo pensaba a que horas me dicen que se murió, yo tenia mucho miedo de esto. De esto si me daba miedo" Madre No 3

Los niños estuvieron hospitalizados en promedio, dos meses, los padres percibieron, que se habían adaptado a la hospitalización de sus hijos, pues los vieron pasar de un estado crítico a la recuperación. Por supuesto los padres experimentaron sentimientos de alegría ante la espectativa de poder llevar a sus niños para la casa, pero también manifestaron temor, pues creían que no contaban con los recursos suficientes para cuidar un pequeño que requería de muchos cuidados. Sin embargo, los padres fueron entrenados por el equipo de salud en los cuidados que debían tener después del alta de los pequeños, en este sentido los participantes manifestaron que las relaciones con el equipo de salud fueron de gran ayuda para ellos, al principio visualizaban a las personas del equipo como obstáculos para la interacción con sus hijos, pero luego esta percepción se fue transformando en confianza, los padres manifestaron que especialmente la enfermera les enseñó múltiples estrategias para el cuidado de sus pequeños.

Finalizando la hospitalización de los niños, los padres tuvieron mayores oportunidades de interacción con sus pequeños, ellos les realizaban el baño, la alimentación... etc., esto constituyó una oportunidad para fortalecer el vínculo afectivo padre-niño, pero también para disminuir el temor de tocarlos y poder empezar a apropiar el rol de cuidadores primarios de sus bebes.
Después de haber vivido múltiples retos debido al estado de salud de los niños, al finalizar la hospitalización, cinco de los participantes fueron informados acerca del diagnóstico de discapacidad visual de sus pequeños y los niños de los otros dos participantes fueron diagnósticados en una consulta de control después de la hospitalización. La noticia les generó gran impacto pues no les habían informado la posibilidad que tenían los niños de padecer ceguera, por lo cual los tomó por sorpresa, ellos pensaban que en ese momento el riesgo de complicaciones de sus niños había pasado y por fin se los llevarían a casa como niños sanos. Dado que el diagnóstico se les informó en momentos distintos, hubo diferencias importantes con relación a las reacciones y sentimientos al rededor del mismo. Los padres de los niños que fueron diagnosticados al final de la hospitalización sintieron dolor, tristeza, angustia, rabia, impotencia, pero fue más fácil que aceptaran porque vivieron simultáneamente el duelo de la prematurez, la hospitalización y el diagnóstico de ceguera, mientras que los padres que conocieron el diagnóstico después de la hospitalización, el proceso de aceptación fue más difícil, aparentemente salieron con un niño sano y el enterarse del diagnóstico de ceguera hizo que vivieran un proceso de angustia, dolor, desesperación, el duelo fue más largo, estos participantes también sintieron disgusto con el equipo de salud, pues les parecía grotesco que hubiese pasado desapercibida tal situación en los niños durante la hospitalización, especialmente si era del conocimiento de ellos que eso pudiera presentarse como una complicación de la prematurez y de la exposición prolongada a oxigenoterapia.

"Cuando lo lleve donde la doctora, me dijo que si al niño antes de darle la salida del hospital le habian hecho todos los exámenes. Ella me dijo el niño como fue prematuro tocaba examinarle los ojos.... Yo estaba muy molesta, pues nunca me dijeron nada" Madre No 4

Hubo diferencias de género en cuanto a las reacciones ante el diagnóstico. El parciticipante mostró tranquilidad, su reacción consistió en salir a documentarse sobre ceguera y se refugió en Dios, esto le ayudó a mantener la esperanza, además buscó información sobre cómo manejaban la discapacidad visual en otros países, lo que le fortaleció la esperanza de un tratamiento curativo para su pequeña. En contraste las madres reaccionaron con llanto, rabia, ellas refirieron sentimientos como incertidumbre, tristeza, vergüenza, dudas, culpa, dolor y miedo. Ellas también refirieron que la fe en Dios fue el principal recurso que tuvieron como estrategia para afrontar esta la situación, creían que un milagro les podría devolver la visión a sus hijos. 


\begin{abstract}
"Nos dio demasiado duro, yo me desmayé. Cuando me dieron la noticia sentí desfallecer, perdí el conocimiento, yo no sabía dónde estaba, no sabía que hacer" Madre No 4
\end{abstract}

Después de darles el diagnóstico seis de los participantes reaccionaron con negación ante la situación, creían que los médicos se habían equivocado, por lo cual consultaron a otros médicos en busca de un diagnóstico diferente o un tratamiento curativo.

Cinco de las participantes presentaron una aceptación parcial frente a la discapacidad visual de sus hijos, se negaron a creer que la ceguera de los niños fuera definitiva y abrigan la esperanza de que la tecnología avance y les ofrezca alguna alternativa curativa para sus hijos. Solo dos participantes lo aceptaron totalmente y continúan adaptándose a los diferentes etapas de la vida de los niños. Sin embargo, todos los participantes expresan que siguen presentando situaciones difíciles, el principal es el glaucoma que presentan varios de los niños, el cual les produce mucho dolor y además el riesgo de una enucleación, lo cual sería para algunos padres vivir un duelo adicional, pues los llevaría a abandonar la esperanza que algún día sus hijos puedan ver.

\section{Viviendo el reto de cuidar al niño en el hogar}

Los participantes manifestaron alegría por tener sus hijos en casa, pero también miedo e inseguridad por no saber cómo cuidarlos, ellos habían recibido instrucciones pero no habían adquirido la suficiente experiencia en el cuidado.

Algunos de los niños necesitaron de cuidados especiales en el hogar, una de la madres debía colocarle una lámpara para brindarle calor y utilizar la técnica de madre canguro, otra debía darle preparados a través de una jeringa, pues la niña no succionaba, otra madre debió continuar la oxigenoterapia en la casa, , esta madre también describió ser muy cuidadosa con la higiene en el hogar como un cuidado importante para la recuperación de su bebé.

Otro aspecto que mostró el estudio, fue que las madres no querían mostrar a sus pequeños a los amigos o vecinos, dejaban a los bebés en su habitación y cuando preguntaban por ellos, decían que continuaban hospitalizados. Sentían verguenza que los vieran, pues los niños eran pequeños, delgados, al ser prematuros los rasgos físicos eran diferentes al de un bebé normal.

Los participantes se dedicaron completamente al cuidado de los niños, disminuyendo el cuidado y la atención hacia sus otros hijos, el de su pareja y el de ellos mismos, una de las participantes no asistía a las citas de control de diabetes, como consecuencia desarrolló falla renal, otra participante no dormía lo suficiente lo que la llevó a una crisis depresiva. El único hombre participante asumió el cuidado de su hija y dejó la responsabilidad económica a su esposa, cambiando su rol de proveedor principal de la familia para encargarse de las labores domésticas.

Los participantes percibieron que era difícil lograr conexión con los niños, sintieron que las caricias, los besos no producían en el pequeño el efecto que los participantes deseaban, como generarles placer, apego por los padres. Poco a poco los participantes fueron creando lazos afectivos a través de un lenguaje táctil y auditivo, que reemplazó el lenguaje visual. Así como los niños crecían, aumentaba la incertidumbre en los padres con relación a cómo sería enseñarles a comer solos, a gatear, a caminar. La principal estrategia que utilizaron los padres fue describir permanentemente las cosas, las situaciones, de donde provenían los sonidos, los colores, las características de las comidas, de las personas, etc. Los padres debieron hacer adaptaciones espaciales de las viviendas para evitar los accidentes en los pequeños, cuando se realizaba algún cambio era informado a los niños, en general las diferentes situaciones familiares giraron en torno a las necesidades del niño.

Finalmente los participantes manifestaron que los niños siempre les enseñaron cosas positivas, los niños vivían con optimismo, con amor, alegría, lo cual dejó en los padres la sensación de que la lucha por sus hijos era pertinente porque los niños les enseñaron a ver la discapacidad como una oportunidad de crecimiento personal.

\section{DISCUSIÓN}

El estudio tuvo como objetivo conocer las experiencias y sentimientos de los padres ante la discapacidad visual de sus hijos. El estudio mostró que los padres de un niño ciego viven múltiples desafíos desde la gestación, la hospitalización y los primeros meses de la crianza del bebé.

Las experiencias estresantes de los padres ante el nacimiento y la subsiguiente hospitalización de un hijo prematuro, está ampliamente descrito en varios estudios $^{13,14,15}$, los autores muestran que los padres de niños hospitalizados en unidades de cuidados intensivos, especialmente si son prematuros, experimentan 
emociones como: incapacidad para proteger al niño, ansiedad y depresión, impotencia, pérdida del control, miedo, incertidumbre y preocupación por las posibles secuelas. Franck y colaboradores ${ }^{15}$ mencionan que la hospitalización del recién nacido produce un choque emocional en los padres, generando sentimientos de incertidumbre y angustia, debido a la no comprensión de los procedimientos a que se ve sometido el pequeño. Estos reportes son similares a los hallazgos del presente estudio.

La pérdida de un niño sano, idealizado y esperado durante la gestación, son aspectos consistentes entre los reportes la literatura ${ }^{17}$ y las experiencias de los participantes de este estudio.

El diagnóstico de ceguera de los niños fue la situación más dolorosa vivida por los participantes; este hallazgo es similar a investigaciones anteriores ${ }^{18}$. Sonksen ${ }^{19}$ considera que la ceguera genera mayor impacto en comparación con otras enfermedades crónicas. Trosterand y Brambring ${ }^{20}$ en su investigación sobre los principios de desarrollo social y emocional de niños ciegos, identificaron que para muchos padres, el nacimiento de un niño ciego es un choque que provoca decepción, tristeza, depresión, ansiedad y culpabilidad. Los padres experimentan incertidumbre porque no saben cómo afrontar el desarrollo cognitivo y emocional del bebé21. En consecuencia son muchas las deficiencias que tienen los padres para el cuidado de estos niños, por lo que los profesionales de la salud, deben determinar cuales son las necesidades que tienen estos padres ${ }^{22,23}$.

Barraga, et al. ${ }^{24}$ muestran que la discapacidad de un hijo genera sentimientos de temor, rechazo, culpa pero raramente se observa amor y aceptación hacia los niños. En contraste, el estudio mostró que los padres experimentación sentimientos como amor, responsabilidad y sobreprotección, pese a que con la discapacidad de sus hijos se vieron alterados los planes que hace todo padre que espera un niño sano.

Los sentimientos de incertidumbre y miedo ante el proceso de crianza de sus hijos con discapacidad visual, experimentados por los participantes, son hallazgos reportados por otros autores ${ }^{19,23}$, éstos muestran que los padres consideran insuficientes sus capacidades 0 recursos para cuidar a un niño con ceguera.

Un estudio ${ }^{21}$ afirma que cada cambio que le sucede al niño con discapacidad visual pone en alerta a la familia, demandando un cambio en los roles, relaciones sociales y en la organización familiar. El cambio en los roles fue vivido por los participantes del presente estudio, desde el momento del diagnóstico, las demandas del cuidado de los niños provocó la disminución del cuidado y la atención a los otros hijos, a su pareja y aún el de ellos mismos, aumentando así, los niveles de angustia y estrés experimentados por los cuidadores.

El rol del profesional de enfermería es fundamental en la ayuda que requieren los padres para afrontar la pérdida de un hijo saludable. Esta ayuda consiste sobre todo en darle información y escucharle. La información no debe ser la tradicional sino enfocada en las estrategias de afrontamiento más efectivas. El padre necesita ser escuchado para que pueda hablar de lo que está viviendo en el presente y cómo cree que será en el futuro. Para el padre puede ser valioso el acercamiento de la enfermera y de los demás profesionales de la salud, y percibir que ellos intentan comprender el impacto de la discapacidad del pequeño en su vida $y$, que están genuinamente interesados en ayudarle a vivir con las limitaciones y exigencias de la discapacidad.

La participación del profesional de enfermería debe iniciar desde el momento en que se determina la gravidez de la mujer, pues las patologías que se presentan durante el embarazo pueden ser evitadas y/o tratadas adecuadamente, para evitar complicaciones tanto a la madre como al niño.

Otro aspecto que debe tenerse en cuenta, con los hallazgos del presente estudio, es la preeminencia del paradigma biologicista en la atención y el cuidado de la diada madre-niño, por lo cual se requiere la introducción en los planes curriculares de contenidos relacionados con el cuidado integral en situación de discapacidad, para que los profesionales de la salud puedan dar respuestas acordes a las necesidades especiales que surgen como consecuencia de la misma.

Es evidente que el apoyo y cuidados del grupo multidisciplinario, especialmente el de la enfermera, podría propiciar una transición menos traumática y mejorar la adaptación de los padres a la discapacidad de sus hijos.

Así, el cuidado de enfermería debe ser un soporte para vivir mejor, utilizando para ello un cuidado integrador, humanizado, que favorece una vida más saludable. Ese cuidado es una acción que se desarrolla y termina en y con la persona, está lleno de valor (ético y estético); es un bien necesario ${ }^{25}$. 


\section{CONCLUSIONES}

La discapacidad visual representa una carga tanto para los niños como sus familias, es un tema que debe ser abordado desde equipos interdisciplinarios dada su complejidad. Para el profesional de enfermería constituye un desafío, pues la meta en la atención y el cuidado, se debe centrar en ayudar a los niños ciegos a utilizar al máximo su capacidad residual, a mantener un óptimo estado de salud y a adaptarse a un estilo de vida moldeado por su condición de discapacidad. El desafío también lo constituye el grupo familiar, pues éste se debe ajustar a su nueva vida, que se modificó a expensas de la discapacidad visual de su niño.

El objetivo principal para los profesionales de salud, es lograr que tanto el niño como su familia vivan un proceso de adaptación a la discapacidad y de inclusión social, lo menos traumático posible.

\section{CONSIDERACIONES ÉTICAS}

El estudio cumplió las normas científicas, técnicas y administrativas para la investigación en salud de la Resolución 008430 de 1993, del Ministerio de Salud de la República de Colombia26. Asimismo, fue aprobado por el comité de Ética de La Facultad de Salud de la Universidad del Valle y del Instituto de Niños Ciegos y Sordos del Valle del Cauca.

\section{CONFLICTO DE INTERÉS}

La autora declara no tener conflictos de interés.

\section{REFERENCIAS}

1. OMS. Discapacidad y salud. Centro de prensa. Nota descriptiva No 352, Diciembre de 2015.

2. Hopenhayn M. Autonomía e independencia: el cuidado de las personas con discapacidad. En: Hopenhayn M, Sojo A, Mancero X. Panorama Social de América Latina. Santiago de Chile; 2012: p. $193-228$

3. Castellanos F, López L. Discapacidad y cultura: desafío emergente en investigación. Avanc Enfermería. 2009; $27(1$ ): 110-123. DOI: 110-123 2346026101214500 .

4. Shrivastava S, Shrivastava P, Ramasamy J. Prevention of Childhood Blindness: Strengthening Primary Health Care. J Ophthalmic Vis Res. 2014; 9(2): 287-288.
5. Kong L, Fry M, Al-Samarraie M, Gilbert C, Steinkuller P. An update on progress and the changing epidemiology of causes of childhood blindness worldwide. J AAPOS. 2012; 16: 501507. DOI: 10.1016/j.jaapos.2012.09.004.

6. Zuluaga C, Sierra M, Asprilla E. Causas de ceguera infantil en Cali, Colombia. Col Médica. 2005; 36 (4): 235-238.

7. Zepeda LC. Causas de Ceguera Infantil. Visión 20/20 Latinoamérica. Boletín Trimestral. Abril 2015

8. Brusi L, Argüello L, Alberdi A, Bergamini J, Toledo F, Mayorga MT, et al. Informe de la salud visual y ocular de los países que conforman la Red Epidemiológica Iberoamericana para la Salud Visual y Ocular (REISVO), 2009 y 2010. Cienc Tecnol Sal Visual Ocular. 2015, 13(1), 11-43. DOI: http://dx.doi.org/10.19052/sv.2961.

9. Morales L, Castillo, E. Lived experiences of adolescents in dialysis: life with multiple loses. Col Médica. 2009; 38(4): Supl 2, 44-53.

10. Martínez C. El muestreo en investigación cualitativa: principios básicos y algunas controversias. Ciênc Saúde Coletiva. 2012; 17(3): 613-619. DOI: http:// dx.doi.org/10.1590/S1413-81232012000300006.

11. Montalvo A, Flórez I, Stavro de Vega D. Cuidando a cuidadores familiares de niños en situación de discapacidad. Aquichán. 2008; 8(2): 197-211.

12. Vásquez ML. Significado da regulaçao da fecundidade para los/as adolescentes numa comunidade urbana marginal. [teses em enfermagen]. Florianópolis: Universidade Federal de Santa Catarina. 2001.

13. Desai P, Jodie B, Bryant S. Care of Children and families in the CICU: A focus on their Developmental, Psychosocial, and Spiritual Needs. Crit Care Nurs Q. 2002; 25(3): 88-97.

14. Myhren H, Ekeberg O, Lagen I, Stoklan O. Emotional strain, communications, and satisfaction of family members in the intensive care unit compared with expectations of the medical staff: experiences from a Norwegian University Hospital. Intensive Care Med. 2004; 30(9): 1791-1798.

15. Lautrette A, Darmon M, Megarbane B, Joly LM, Chevret S, Adrie C, et al. A Comunication Strategy and Brochure for Relatives of Patients Dying in the ICU. N Engl J Med. 2007; 356(5): 469-478.

16. Franck L, Cox S, Allen A, Winter I. Parental concern and distress about infant pain. Archives of Disease in Childhood Fetal and Neonatal Edition. 2004. 89(1): 71-75.

17. Frank L, Cox S, Allen A, Winter I. Measuring neonatal intensive care unit-related parental stress. J Adv Nurs. 2005; 49 (6): 608-615. 
18. Freeman R, Goetz E, Richards P, Groenveld M, Blockberger S, Jan JE, et al. Blind children's early emotional development: do we know enough to help?. Child Care Health Dev. 1989; 15(1): 3-28. DOI: $10.1111 / \mathrm{j} .1365-2214.1989 . t b 00596 . x$.

19. Sonksen P. The assement of "Vision for development" in severely visually handicapped babies. Acta Ophthalmol Suppl. 1983; 61(157): 82-90.

20. Troster H, Brambring M. Early social-emotional development in blind infants. Child Care Health Dev. 1992; 18(4): 207-227.

21. Checa J, Díaz P, Pallero R. Psicología y ceguera. Manual para la intervención psicológica en el ajuste a la discapacidad visual. Organización Nacional de Ciegos Españoles. 1a ed. Madrid: Manuales; 2003 p 30-61, 243-259.

22. Behl D, Akers J, Boyce M, Taylor M. Do mothers interact differently with children who are visually impaired? JVIB. 1996; 90: 501-512.

23. Leonhardt M. Colección de Psicopedagogía y Lenguaje. El bebe ciego. Primera atención. Un enfoque psicopedagógico. Barcelona: Masson S. A. 1992.

24. Barraga N, Mangold S, Neff J. Discapacidad Visual III, International council for education of the visually handicapped christotoffel Blindenmission, Región Latinoamericana. 1986. Argentina. 48: 1-43.

25. Rocha P, Prado M, Lenise M, de Gasperri P, Sebold L, Waterkemper R, et al . Avances en Enfermería. 2009; 27(1).

26. Resolución 8430 de 1993. Por la cual se establecen las normas científicas, técnicas y administrativas para la investigación en salud. Ministerio de Salud de Colombia. Octubre 4 de 1993. 